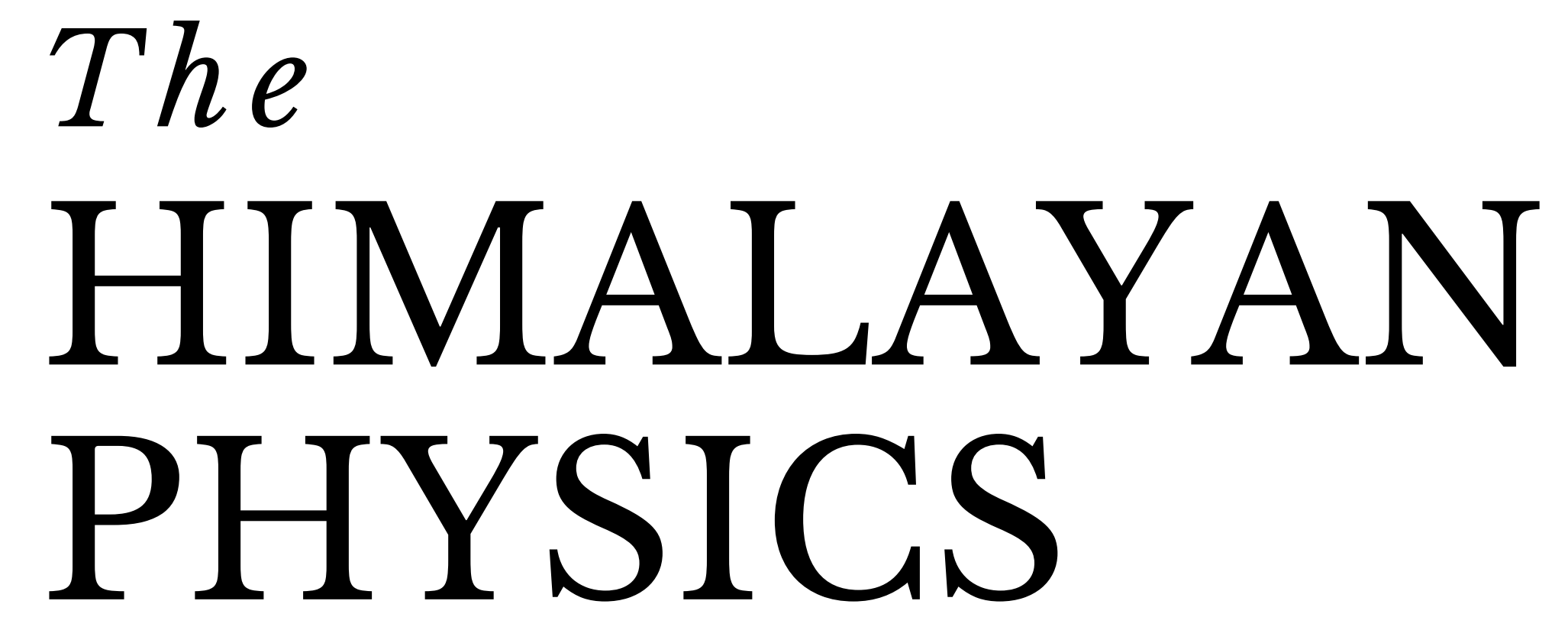

A peer-reviewed Journal of Physics

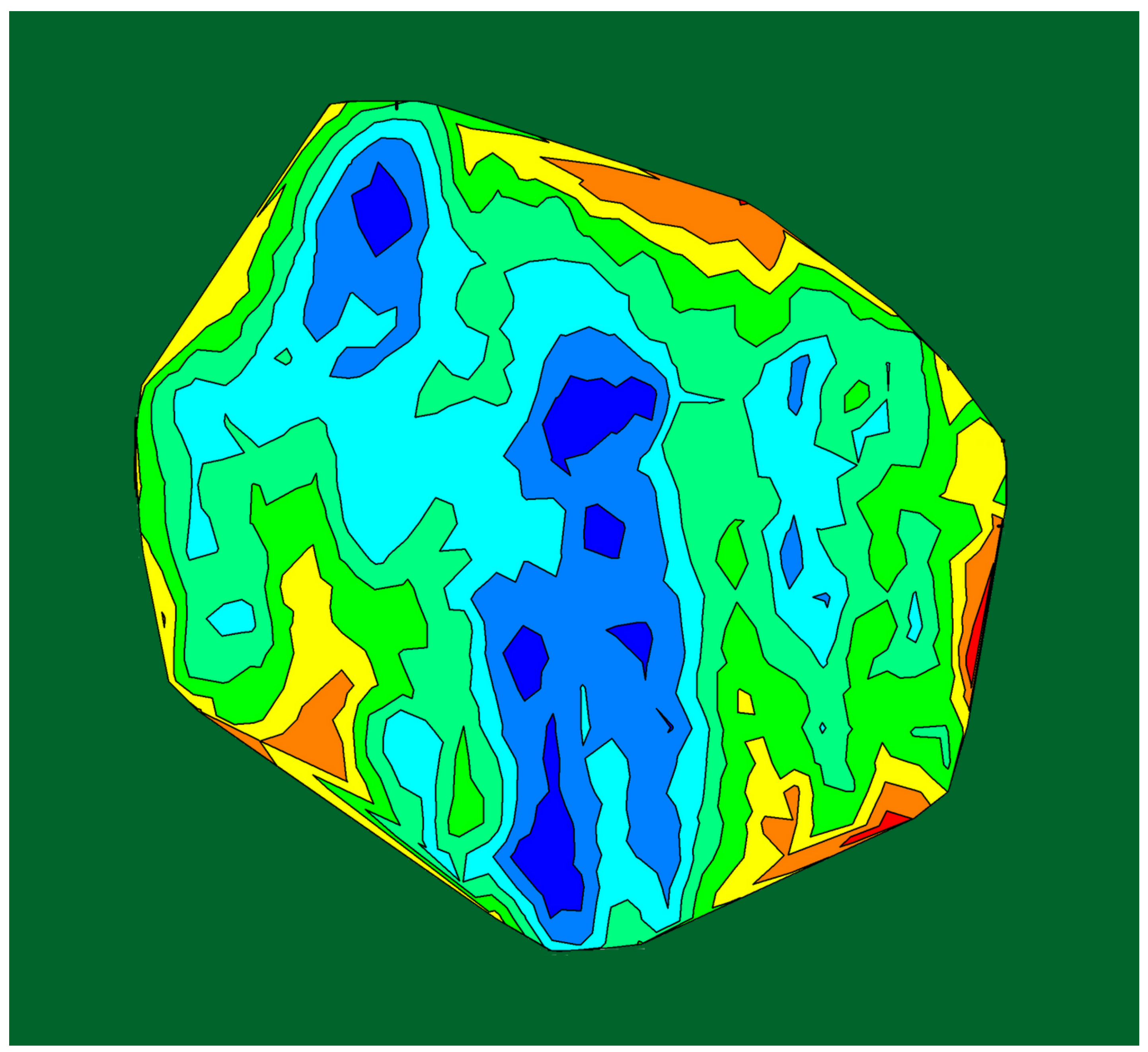

Department of Physics, Prithvi Narayan Campus, Pokhara Nepal Physical Society, Western Chapter, Pokhara 


\section{Publisher}

Department of Physics, Prithvinarayan Campus, Pokhara Nepal Physical Society, Western Chapter, Pokhara

\section{The Himalayan Physics}

Volume 9, December 2020

ISSN 2542-2545

The Himalayan Physics (HimPhys) is an open access peer-reviewed journal that publishes quality articles which make innovative contributions in all areas of Physics. HimPhys is published annually by Nepal Physical Society (Western Regional Chapter), and Department of Physics, Prithvi Narayan Campus, Pokhara. The goal of this journal is to bring together researchers and practitioners from academia in Nepal and abroad to focus on advanced techniques and explore new avenues in all areas of physical sciences and establishing new collaborations with physics community in Nepal.

\section{Chief Editor}

Kapil Adhikari

\section{Associate Editor \\ Aabiskar Bhusal}

(C)2020, Publishers. All rights reserved.

This publication is in copyright. Subject to statutory exception and to the provisions of relevant collective licensing agreements, no reproduction of any part may take place without written permission of the publishers.

Cover: Contour map of dust mass. (C) Mijas Tiwari. Printed from article in the current issue, with permission. 


\section{The HIMALAYAN PHYSICS}

A peer-reviewed Journal of Physics

\section{Chief Editor}

Kapil Adhikari

Associate Editor

Aabiskar Bhusal

\section{Publisher}

Department of Physics, Prithvi Narayan Campus, Pokhara Nepal Physical Society, Western Chapter, Pokhara 


\section{Nepal Physical Society Western Regional Chapter Pokhara, Nepal}

\section{President}

Min Raj Lamsal

Immediate Past President

Jeevan Regmi

Vice-President

Sundar Prasad Dhakal

Secretary

Ravi Karki

Treasurer

Dipak Adhikari

Joint Secretary

Sujan Lamsal

Editorial Member

Kapil Adhikari

Members

Amrit Dhakal

Laxman Thapa

Laxman Timilsina

Narayan Prasad Bhandari

Pradeep Subedi

Advisory Board

Prof. Dr. Pradip K. Bhattarai

Pabitra Mani Poudyal

Surya Bahadur G.C.

Parashu Ram Poudel

Prof. Dr. Shovakanta Lamichhane

Kul Prasad Dahal

Dr. Krishna Raj Adhikari

Ram Sajile Verma 


\section{Himalayan Physics Vol-9 (2020)}

\section{TABLE OF CONTENTS}

Metal Organic Frameworks(MOFs) as efficient carrier for targeted nanodrug delivery

R. Karki, D. Adhikari, K. Adhikari, N. Pantha

A Density Functional Theory Study on Paracetamol-Oxalic Acid Co-Crystal

P. Paudel, K.R. Adhikari, K. Adhikari

First-principles study of $\mathrm{C}$ cites vacancy defects in water adsorbed graphene

H.K. Neupane, N.P. Adhikari

Diusion of fructose in water: a molecular dynamics study

S. Bhusal, N. Pantha

Study of aecting factors of meteorological parameters on solar radiation on Pokhara P.M. Shrestha, J. Regmi, U. Joshi, K.N. Poudyal, N.P. Chapagain, I.B. Karki

Variation of mean value of velocity of ion with dierent obliqueness of magnetized plasma sheath

B.R. Adhikari, H.P. Lamichhane, R. Khanal

Study of dust properties of two far infrared cavities nearby asymptotic giant branch stars under infrared astronomical satellite maps

M. Tiwari, S.P. Gautam, A. Silwal, S. Subedi, A. Paudel, A. K. Jha

An experimental study on irradiated interface of silicon

M.R. Lamsal

Calculation of energy loss of proton beam on thyroid tumor

K. Giri, B. Paudel, B.R. Gautam

Study of noise level status at dierent rice mills in Surkhet Valley, Nepal

D.R. Paudel, H.N. Baral

Elliptically polarized laser assisted elastic electron-hydrogen atom collision and differential scattering cross-section

K. Yadav, S.P. Gupta, J.J. Nakarmi

Geodynamics of Gorkha earthquake (Mw 7.9) and its aftershocks

R.K. Tiwari and H. Paudyal 


\title{
Metal Organic Frameworks (MOFs) as efficient carrier for targeted nanodrug delivery
}

Review Article

\author{
Ravi Karki $^{1}{ }^{*}$, Dipak Adhikari $^{1}{ }^{\dagger}$, Kapil Adhikari $^{1,2}$, Nurapati Pantha ${ }^{3}$ \\ 1 Department of Physics, Prithvi Narayan Campus, Pokhara, Nepal \\ 2 Physics Research Initiatives (PRI), Pokhara, Nepal \\ 3 Central Department of Physics, Tribhuvan University, Nepal
}

\begin{abstract}
A controllable and targeted drug delivery system development is imperative and important to reduce side effects and enhance the therapeutic efficacy of drugs. Metal-organic frameworks (MOFs), an emerging type of hybrid porous materials synthesized from metal ions or clusters abridged by organic linkers. They have attracted increasing attention in the recent years owing to the unique physical structures possessed, and the potential for wide range of applications. The superior properties of MOFs, such as well-defined pore aperture, tailorable composition and structure, tunable size, versatile functionality, high agent loading, and improved biocompatibility, have made them promising candidates as drug delivery hosts. MOFs for drug encapsulation and delivery is of great interest and many very promising results have been found, indicating that these porous solids exhibit several advantages over existing systems. This review highlights the recent advances in the synthesis, functionalization, and applications of MOFs in nanodrug delivery, and has classified them using drug loading strategies.

Keywords: Metal Organic Frameworks • Active Pharmaceutical Ingredients $\bullet$ Nanodrug delivery $\bullet$ pH responsive drug delivery
\end{abstract}

\section{Introduction}

Metal Organic Frameworks (MOFs),an emerging type of hybrid porous materials constructed from metal ions abridged by organic ligands or linkers have attracted increasing attention in recent years. The versatile and superior properties of MOFs, excessively large surface area, well defined porosity, tailorable composition and structure, tunability in size, versatile functionality, high loading agent, intrinsic biodegradability and enhanced biocompatibility, raised MOFs as promising candidates in multidimensional disciplines [1]. Researchers have shown the efficacy of MOFs in various fields as clean energy [2], heterogeneous catalysis [3], biomedical imaging [4], sensing [5], drug delivery [6], water captures [7] nerve agent destructions [8],toxic chemical removals [9], water purification [10], gas storage [11], gas separation [12], magnetic resonance imaging [13], computed tomography

\footnotetext{
* Corresponding Author: rabi.karki@prnc.tu.edu.np

$\dagger$ Corresponding Author: dipak.adhikari@prnc.tu.edu.np
} 
[14], optical imaging [15] etc. The status of commercialization of MOF-based products has broad scope in the future directions. On fundamental level, MOFs epitomize the beauty of chemical structures and the power of combining organic and inorganic chemistry. Since 1990s, the MOF field has experienced an almost unparalleled growth, evidenced not only by the enormous number of research papers published but also by the ever-expanding scope of the research field .

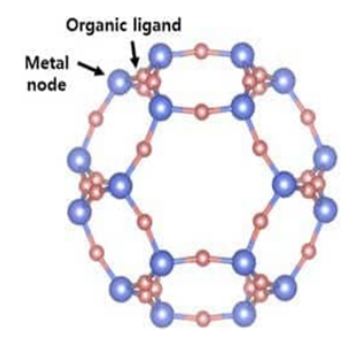

Figure 1. A sample of MOF [16]

\section{Drug loading in MOFs}

Despite remarkable progress in our understanding of the fundamental biology behind many diseases, we have yet to observe the comparable advances in the drug delivery of these diseases. Current therapeutics are limited by their nonspecific distribution throughout the body leading to high doses, rapid clearance, poor pharmacokinetics and high side effects.

While a number of strategies have been developed to improve the efficacy of conventional drugs, nanoparticle based therapeutics have received an increasing amount of attention over last two decades [18]. Some of the important properties of nanomaterials are small sized, high drug loading, surface properties, drug release kinetics, improved pharmacokinetics, biodegradability and biocompatibility. Moreover, nanoparticles can be specifically targeted to the diseased tissues (tumors) by conjugation with ligands and can be engineered to contain multiple agents such as imaging and therapy which is impossible to achieve in conventional therapeutics [19].

MOFs exhibit many desired characteristics as drug careers including exceptionally high surface areas and large pore sizes for the drug encapsulation, intrinsic biodegradability as a result of relatively labile metal-ligand bonds and versatile functionality for post synthetic grafting of drug molecules. Over the past decade, MOFs have been investigated for applications in loading of and release of several drug molecules [20]. They are scale downed to nanoregime to form nanoscale metal-organic frameworks (NMOFs) by using variety of different techniques that have been developed for inorganic and organic polymeric nanoparticles [21]. 


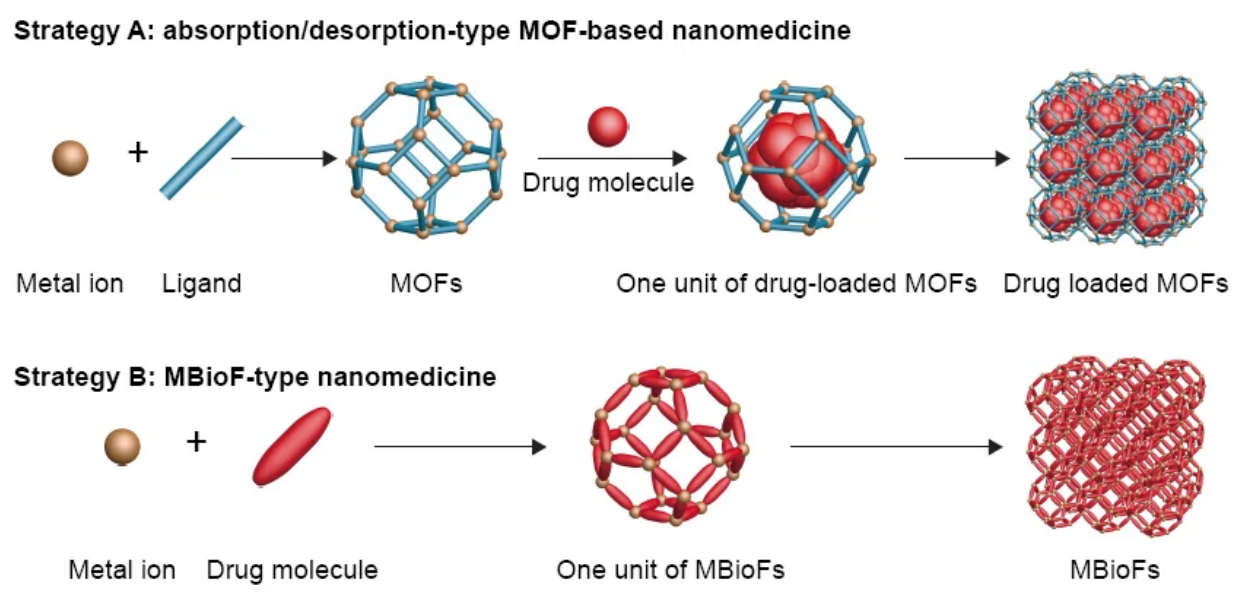

Figure 2. Formation of absorption/desorption-type MOF nanomedicine and MBioF-type nanomedicine by coordination-directed self-assembly process [4]

NMOFs are potential nanovehicles for delivering therapeutic agents to the targeted area of the body as they are able to control drug release, with their large surface areas, high porosity and the presence of functional groups to interact with the loaded moieties [22].

\section{The first experimental studies of MOFs as potential drug carriers}

In 2006, Horcajada et al. investigated the first MOF family (Table 1) that can be used as potential drug carriers [23]. The two MOFs studied, MIL-100 and MIL-101 (MIL is the abbreviation of Materials of Institute Lavoisier), are composed of trivalent metal centers with carboxylic linkers and possess large pores with large surface areas. These structural characteristics promote the use of MILs as efficient drug carriers. They carried out experiments to study the loading and release kinetics of the analgesic drug, Ibuprofen (IBU), in both MIL structures. The loading capacity of IBU in MIL-100 reached $0.347 \mathrm{~g}$ IBU per g MOF and in MIL-101 reached $1.376 \mathrm{~g}$ IBU per g MOF. The difference was attributed to the volume of each cage for MIL-101 $\left(12700 \AA^{3}\right.$ and $20600 \AA^{3}$ ) and for MIL-100 (8200 $\AA^{3}$ and $12700 \AA^{3}$ ). The Rosi group created a MOF consisting of zinc(II) ions, adenine, and parabiphenyl-dicarboxylic acid [22]. It was loaded with a hydrochloride salt of the antiarrhythmic procainamide (PROC). The complete loading (0.22 g PROC per g MOF) was achieved after 15 days, and a steady cation-triggered PROC release was observed within $20 \mathrm{~h}$, while a complete release was observed after 72 h. Sun et al. achieved the highest loading of $50 \mathrm{wt} \%$ of the antitumor agent 5-FU using a $\mathrm{Zn}-\mathrm{MOF}$ containing an achiral ligand [24]. Furthermore, other biocompatible MOFs have also been studied as promising materials for drug storage and controlled drug release. A few examples of such applications and loading capacity of the mentioned MOFs are presented below in Table 1. 
Table 1. : Drugs encapsulated in different MOF [25]

\begin{tabular}{c|c|c|c}
\hline MOF & Drug & Loading & $\begin{array}{c}\text { Application } \\
\%\end{array}$ \\
\hline MIL-100(Cr) & Ibu & 35 & $\begin{array}{c}\text { Anti- } \\
\text { inflammatory/analgesic }\end{array}$ \\
\hline HKUST- & Nimesulide & 20 & \\
\hline $1(\mathrm{Cu})$ & & & \\
\hline MIL-101(Cr) & Ibu & 1.38 & Anti-inflammatory \\
\hline MIL-100(Fe) & ESCP & 13 & Antitumoral \\
\hline MIL-100(Fe) & Doxo & 9 & \\
\hline MIL-100(Fe) & Bu & 25 & \\
\hline Zn(bix $)$ & Camptothecin & 21 & \\
\hline Zn(bix $)$ & Daunomycin & 21 & \\
\hline ZIF-8(Zn) & 5 -FU & 45 & \\
\hline MIL-100(Fe) & CDV & 16 & Antiviral \\
\hline MIL-100(Fe) & AZT-Tp & 24 & Antiretroviral \\
\hline
\end{tabular}

The experimental study of DOX loading experiments were performed using the standard protocols [26]. At first, the carrier i.e. MF (or MNFx) was dried at $100^{\circ} \mathrm{C}$ for about $4 \mathrm{~h}$. About $110 \mathrm{mg}$ of this dried carrier was added to $15 \mathrm{ml}$ of as purchased DOX solution and kept under dark in an orbital shaker at 200 rpm and $25^{\circ} \mathrm{C}$ for about $24 \mathrm{~h}$. The sample was recovered in a high speed centrifuge (run at 10,000 rpm for about 10 min). The entrapment efficiency and the drug loading capacity of the carrier were calculated from the standard equations [24]. The DOX release experiments were carried out in the phosphate buffer saline (PBS) solution of pH 7.4. Typically, for making of 1L PBS, following reagents $\mathrm{NaCl}(8.0 \mathrm{~g}), \mathrm{KCl}(0.2 \mathrm{~g}), \mathrm{Na}_{2} \mathrm{HPO}_{4}(1.42 \mathrm{~g})$ and $\mathrm{KH}_{2} \mathrm{PO}_{4}(0.24 \mathrm{~g})$ were dissolved in $800 \mathrm{ml}$ of water. The $\mathrm{pH}$ of the solution was measured as 7.4 [24]. The DOX release experiments were carried out successfully by the protocol similar to that described in literature [25, 26]. The release profile was also obtained by measuring the supernatant concentration in the PBS at periodic time intervals [27].

\section{Some other successful results of drug loadings}

Ilknur Erucara and Seda Keskinb [24] examined the potential of ten different MOF-74 materials as binary drug carrier systems. Understanding simultaneous adsorption and diffusion of anticancer drug molecules in the host material is important to develop combination therapy for cancer. They performed Configurational Bias Monte Carlo and Molecular Dynamics simulations to compute storage and diffusion of two anticancer drugs, methotrexate (MTX) and 5-fluorouracil (5-FU), in MOFs. RAVXIX was identified as the top performing MOF which has the highest MTX $(2.8 \mathrm{~g} / \mathrm{g})$ and 5 -FU $(4.2 \mathrm{~g} / \mathrm{g})$ uptakes due to its extremely large pore volume. They showed strong linear relations between pore volumes/pore sizes and drug uptake capacities of MOFs. Both single-component and binary adsorption isotherms of MTX and 5-FU molecules were computed in four MOF- 
74 materials. Results showed that RAVXET, RAVWUI, RAVXAP and RAVXIX can be potential materials for storage of MTX and 5-FU mixtures. Also they examined single-component and mixture diffusion of MTX and 5-FU molecules through the pores of MOFs using MD simulations. Slow diffusion of drug molecules in MOF-74 materials suggested that MOFs have strong potential as multi-drug carrier systems for combination therapies in cancer research. The computational methodology that we use in this work will be useful to identify effectively the most promising MOFs for target drug storage and delivery applications prior to extensive experiments. By providing fundamental insights at the molecular-level on the drug adsorption and diffusion mechanism of MOFs, this study will also guide the design and development of new MOFs as multi-drug delivery systems.

\section{Material stability and drug release}

If a $\mathrm{MOF}$ is intended as a drug delivery system in biomedical filed, especially in cancer treatment, the material has not only to adsorb a high amount of drug, but also to release it and the release should be preferably in a controlled manner. Optimally, the drug release should take place completely in an acidic pH often found in tumorous tissues (often around 6.0), while being as low as possible in the pH range of blood and healthy cells (around 7.4) [28]. Therefore, both material stability and drug release were studied in these two pH values. The material stability was studied by dispersing the As (III) drug loaded material in a phosphate buffered saline of two different $\mathrm{pH}$ values -6.0 and 7.4. The material was kept in the solution at $37^{\circ} \mathrm{C}$ for $4,24,72$ and $168 \mathrm{~h}$. At $\mathrm{pH}$ 7.4 the MOF was at least moderately stable for the first 4 hours, whereas at pH 6.0 the MOF decomposed quite rapidly, and could not be detected it after the first 4 hours by any of the two methods. Noticeably, the crystalline phase of the phosphate salt was firstly detected after 3 days, suggesting that the material firstly transformed into an amorphous phase before the zinc ions were released. This was also confirmed by ICP_OES images of the nanoparticles taken after $24 \mathrm{~h}$ of the drug release studies (figure 5).

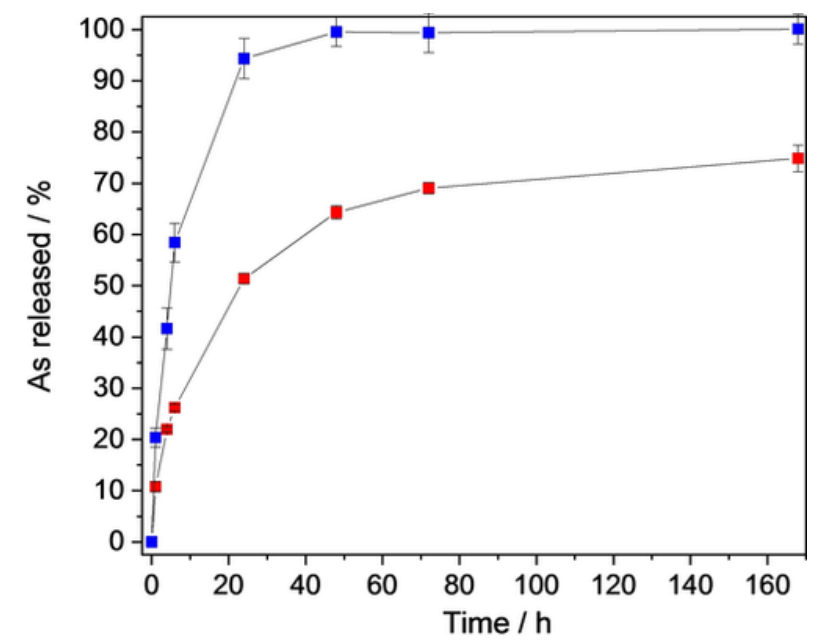

Figure 3. Arsenic release from the drug loaded Zn-MOF-74 at pH 6.0 (blue) and 7.4 (red) at $37^{\circ} \mathrm{C}$ in phosphatebuffered saline, determined by ICP-OES [29] 
To evaluate the drug release, the As (III) drug loaded Zn MOF-74 nanoparticles were dispersed in phosphate buffered saline of two $\mathrm{pH}$ values (6.0 and 7.4) and kept in the solution at $37 \mathrm{degC}$. The amount of arsenic and zinc ions released into the solution was determined after 1, 4, 6, 24, 48, 72 and $168 \mathrm{~h}$ by ICP_OES. The findings of the release studies were in agreement with the stability data reported for Zn MOF-74 [30]. In the study, the material stability was investigated as a function of vapour pressure and temperature. Therefore, in a lower pH where the concentration of protons is higher, a faster material decomposition is expected. To fine tune the material stability further and also for biocompatibility, surface modification including polymer coating is possible [31]. The results of the arsenic release studies clearly showed that Zn MOF-74 could be used as a drug carrier for pH triggered drug delivery. Additionally, in comparison to the two previously reported MOFs for arsenic trioxide delivery: MFU 4I (drug loading: $237 \mathrm{mg}$ of $\mathrm{A}_{2} \mathrm{O}_{3} / 1 \mathrm{~g}$; not $\mathrm{pH}$ responsive) [15] and $\mathrm{ZIF} 8$ (drug loading: $98 \mathrm{mg}$ of $\mathrm{As}_{2} \mathrm{O}_{3} / 1 \mathrm{~g} ; \mathrm{pH}$ responsive) [32], it combines the unique properties of both systems-it exhibited high drug loading capacity (153 $\mathrm{mg}$ of $\mathrm{As}_{2} \mathrm{O}_{3} / 1 \mathrm{~g}$ ) and features $\mathrm{pH}$-triggered drug release.

\section{Recent Researches on Drug Delivery in MOFs}

Very recently a number of research works are carried out in drug delivery through MOFs. Some of them are described here. Weicong Liu and et al [33] study explored the high storage capacities and excellent biocompatibilities of zinc metal-organic frameworks (Zn-MOFs). Their study on the pH-responsive processes based on carrier-drug interactions have proven them to be the most efficient and effective way to control the release profiles of drugs. Also, the perspectives and future challenges in this type of carrier system are discussed.

The various methods for modifying the surfaces of Zr-MOFs were described with pertinent examples of the resulting enhancements in aqueous stability, colloidal dispersion, and stimuli-responsive drug release [34]. The in vitro and in vivo application of Zr-MOFs for photodynamic therapy and drug delivery were discussed with respect to the structural features of the MOFs and their surface functionality, and perspectives on their future applications. Experimentally and computationally it was verified that doxorubicin can be pore loaded in MIL-101(Cr) and surface loaded on UiO-66, different time-dependent cytotoxicity profiles are observed by real-time cell analysis and confocal microscopy [35]. The attenuated release of aggregated doxorubicin from the surface of Dox@UiO-66 results in a 12 to $16 \mathrm{~h}$ induction of cytotoxicity, while rapid release of pore-dispersed doxorubicin from Dox@MIL101(Cr) leads to significantly higher intranuclear localization and rapid cell death.

It was reported, the use of CAU-7, a biocompatible bismuth-based MOF, for the delivery of two cancer drugs, sodium dichloroacetate (DCA) and $\alpha$-cyano-4-hydroxycinnamic acid ( $\alpha$-CHC). Loadings of 33 and 9 wt $\%$ for DCA and $\alpha$-CHC, respectively are achieved. Interestingly, CAU-7 showed a gradual release of the drugs, achieving a release time of up to 17 days for DCA and 31 days for $\alpha$-CHC [36]. With the thermal treatment, it was able to achieve an outstanding 32\% slower release of $\alpha$-CHC from the thermally treated CAU-7. Using in vitro studies and endocytosis inhibitors, confocal microscopy, and fluorescence-activated cell sorting, it was demonstrated that 
CAU-7 was successfully internalized by cancer cells, partially avoiding lysosome degradation. Finally, it was found that CAU-7 loaded either with DCA or $\alpha$-CHC had a higher therapeutic efficiency compared with the free drug approach, making CAU-7 a great option for biomedical applications.

The antibacterial action of nanomaterials offers major modalities to combat drug resistance of bacteria. It was reported that the use of nano-metal-organic frameworks on encapsulating drug molecules enhances its antibacterial activity against model drug-resistant bacteria and biofilm of the bacteria. Rifampicin (RF), a well-documented antituberculosis drug with tremendous pharmacological significance, was attached into the pore surface of zeolitic imidazolate framework 8 (ZIF8) by a simple synthetic procedure. The drug release profile experiment demonstrated that ZIF8-RF depicts pH-responsive drug delivery and is ideal for targeting bacterial disease corresponding to its inherent acidic nature [37]. Most remarkably, ZIF8-RF gave enhanced antibacterial activity against methicillin-resistant Staphylococcus aureus bacteria and also prompts entire damage of structurally robust bacterial biofilms. Overall, the study depicted a detailed physical insight for manufactured antibiotic-encapsulated NHs presenting tremendous antimicrobial activity that can be beneficial for manifold practical applications.

In the recent decade, a large number of experimental works and researches on drug delivery through MOFs are performed but very few theoretical studies and researches are carried out in this field comparatively. Here we use different theoretical and computational approaches to explore the possibility of different active pharmaceutical ingredients on different types MOF-74.

\section{Future Scope}

The knowledge about metal organic frameworks is growing rapidly during recent few years but there are still significant gaps in the completeness of our understanding of their structure, stability and properties. Detailed investigations on factors responsible for destruction of crystal structure of certain MOFs with time stability and decomposition mechanism have still not been carried out systematically. There are some reports on applications

of MOFs in drug loading and delivery for some anticancer and antiviral molecules. MOFs can be explored for such application for other drug molecules too. Detailed toxicological investigation should be carried out commercialization of such products. 


\section{References}

[1] Wu MX, Yang YW. Metal-organic framework (MOF)-based drug/cargo delivery and cancer therapy. Advanced Materials. 2017;29(23):1606134.

[2] Emam HE, Abdelhameed RM, Ahmed HB. Adsorptive performance of MOFs and MOF containing composites for clean energy and safe environment. Journal of Environmental Chemical Engineering. 2020;p. 104386.

[3] Nguyen LT, Nguyen TT, Nguyen KD, Phan NT. Metal-organic framework MOF-199 as an efficient heterogeneous catalyst for the aza-Michael reaction. Applied Catalysis A: General. 2012;425:44-52.

[4] Della Rocca J, Liu D, Lin W. Nanoscale metal-organic frameworks for biomedical imaging and drug delivery. Accounts of chemical research. 2011;44(10):957-968.

[5] Kreno LE, Leong K, Farha OK, Allendorf M, Van Duyne RP, Hupp JT. Metal-organic framework materials as chemical sensors. Chemical reviews. 2012;112(2):1105-1125.

[6] Sun CY, Qin C, Wang XL, Su ZM. Metal-organic frameworks as potential drug delivery systems. Expert opinion on drug delivery. 2013;10(1):89-101.

[7] Rieth AJ, Yang S, Wang EN, Dincă M. Record atmospheric fresh water capture and heat transfer with a material operating at the water uptake reversibility limit. ACS central science. 2017;3(6):668-672.

[8] Peterson GW, Moon SY, Wagner GW, Hall MG, DeCoste JB, Hupp JT, et al. Tailoring the pore size and functionality of $\mathrm{UiO}$-type metal-organic frameworks for optimal nerve agent destruction. Inorganic chemistry. $2015 ; 54(20): 9684-9686$.

[9] Moghadam PZ, Fairen-Jimenez D, Snurr RQ. Efficient identification of hydrophobic MOFs: application in the capture of toxic industrial chemicals. Journal of Materials Chemistry A. 2016;4(2):529-536.

[10] Hasan Z, Jhung SH. Removal of hazardous organics from water using metal-organic frameworks (MOFs): plausible mechanisms for selective adsorptions. Journal of hazardous materials. 2015;283:329-339.

[11] Li H, Wang K, Sun Y, Lollar CT, Li J, Zhou HC. Recent advances in gas storage and separation using metal-organic frameworks. Materials Today. 2018;21(2):108-121.

[12] Li JR, Kuppler RJ, Zhou HC. Selective gas adsorption and separation in metal-organic frameworks. Chemical Society Reviews. 2009;38(5):1477-1504.

[13] Chowdhury MA. M etal-organic-frameworks for biomedical applications in drug delivery, and as MRI contrast agents. Journal of Biomedical Materials Research Part A. 2017;105(4):1184-1194.

[14] Dekrafft KE, Boyle WS, Burk LM, Zhou OZ, Lin W. Zr-and Hf-based nanoscale metal-organic frameworks as contrast agents for computed tomography. Journal of materials chemistry. 2012;22(35):18139-18144.

[15] Gao X, Zhai M, Guan W, Liu J, Liu Z, Damirin A. Controllable synthesis of a smart multifunctional nanoscale metal-organic framework for magnetic resonance/optical imaging and targeted drug delivery. ACS applied materials \& interfaces. 2017;9(4):3455-3462. 
[16] Soni S, Bajpai PK, Arora C. A review on metal-organic framework: synthesis, properties and application; 2018. .

[17] Mendes RF, Figueira F, Leite JP, Gales L, Paz FAA. Metal-organic frameworks: a future toolbox for biomedicine? Chemical Society Reviews. 2020;.

[18] Desai N. Challenges in development of nanoparticle-based therapeutics. The AAPS journal. 2012;14(2):282295.

[19] Langer R. New methods of drug delivery. Science. 1990;249(4976):1527-1533.

[20] Dong Z, Sun Y, Chu J, Zhang X, Deng H. Multivariate metal-organic frameworks for dialing-in the binding and programming the release of drug molecules. Journal of the American Chemical Society. 2017;139(40):14209-14216.

[21] Beg S, Rahman M, Jain A, Saini S, Midoux P, Pichon C, et al. Nanoporous metal organic frameworks as hybrid polymer-metal composites for drug delivery and biomedical applications. Drug Discovery Today. 2017;22(4):625-637.

[22] Huxford RC, Della Rocca J, Lin W. Metal-organic frameworks as potential drug carriers. Current opinion in chemical biology. 2010;14(2):262-268.

[23] Horcajada P, Serre C, Vallet-Regí M, Sebban M, Taulelle F, Férey G. Metal-organic frameworks as efficient materials for drug delivery. Angewandte chemie. 2006;118(36):6120-6124.

[24] Erucar I, Keskin S. Computational investigation of metal organic frameworks for storage and delivery of anticancer drugs. Journal of Materials Chemistry B. 2017;5(35):7342-7351.

[25] Tamames-Tabar C, García-Márquez A, Blanco-Prieto M, Serre C, Horcajada P, Ruiz-Molina D, et al.. Bio-and bioinspired nanomaterials. Wiley-VCH Verlag GmbH \& Co. KGaA; 2015.

[26] Bhattacharjee A, Gumma S, Purkait MK. Fe3O4 promoted metal organic framework MIL-100 (Fe) for the controlled release of doxorubicin hydrochloride. Microporous and Mesoporous Materials. 2018;259:203-210.

[27] Bhattacharjee A, Purkait MK, Gumma S. Doxorubicin loading capacity of MIL-100 (Fe): effect of synthesis conditions. Journal of Inorganic and Organometallic Polymers and Materials. 2020;30(7):2366-2375.

[28] Tannock IF, Rotin D. Acid pH in tumors and its potential for therapeutic exploitation. Cancer research. $1989 ; 49(16): 4373-4384$.

[29] Schnabel J, Ettlinger R, Bunzen H. Zn-MOF-74 as pH-responsive drug-delivery system of arsenic trioxide. ChemNanoMat. 2020;6(8):1229-1236.

[30] Tan K, Zuluaga S, Gong Q, Canepa P, Wang H, Li J, et al. Water reaction mechanism in metal organic frameworks with coordinatively unsaturated metal ions: MOF-74. Chemistry of Materials. 2014;26(23):68866895.

[31] Forgan RS. The surface chemistry of metal-organic frameworks and their applications. Dalton Transactions. 2019;48(25):9037-9042.

[32] Yaghi OM, O’Keeffe M, Ockwig NW, Chae HK, Eddaoudi M, Kim J. Reticular synthesis and the design of 
new materials. Nature. 2003;423(6941):705-714.

[33] Liu W, Pan Y, Xiao W, Xu H, Liu D, Ren F, et al. Recent developments on zinc (ii) metal-organic framework nanocarriers for physiological pH-responsive drug delivery. MedChemComm. 2019;10(12):2038-2051.

[34] Lazaro IA, Forgan RS. Application of zirconium MOFs in drug delivery and biomedicine. Coordination Chemistry Reviews. 2019;380:230-259.

[35] Markopoulou P, Panagiotou N, Li A, Bueno-Perez R, Madden D, Buchanan S, et al. Identifying differing intracellular cargo release mechanisms by monitoring in vitro drug delivery from MOFs in real time. Cell Reports Physical Science. 2020;1(11):100254.

[36] Orellana-Tavra C, Köppen M, Li A, Stock N, Fairen-Jimenez D. Biocompatible, crystalline, and amorphous bismuth-based metal-organic frameworks for drug delivery. ACS applied materials \& interfaces. 2020;12(5):5633-5641.

[37] Ahmed SA, Nur Hasan M, Bagchi D, Altass HM, Morad M, Althagafi II, et al. Nano-MOFs as targeted drug delivery agents to combat antibiotic-resistant bacterial infections. Royal Society Open Science. 2020;7(12):200959. 